\title{
Coccygeal Movement Test: An Objective, Non-Invasive Test for Localization of the Pelvic Floor Muscles in Healthy Women
}

\author{
Stine H. Stensgaard ${ }^{a}$ Karl Moeller Bek ${ }^{b}$ Khaled M.K. Ismail ${ }^{c, d}$ \\ ${ }^{a}$ Perinatal Research Unit and ${ }^{b}$ Department of Obstetrics and Gynecology, Aarhus University Hospital, Skejby, \\ Denmark; ' ${ }^{C}$ Birmingham Centre for Women and Children Health, School of Clinical and Experimental Medicine, \\ College of Medical and Dental Sciences, University of Birmingham, and 'Academic Unit, Birmingham Women's NHS \\ Foundation Trust, Birmingham, UK
}

\section{Key Words}

Pelvic floor · Incontinence - Coccygeal movement test .

Pelvic floor muscle training

\section{Abstract}

Objective: To evaluate the use of palpation of the coccyx (the coccygeal movement test, CMT) as a possible objective screening tool for the assessment of adequate localization of the pelvic floor muscles (PFMs). Subjects and Methods: Twenty-four healthy female volunteers, known to be able to locate their PFMs, were given instructions (allocated at random) to perform a 'correct contraction', 'straining' or 'nothing' when examined by six independent assessors using the CMT. The assessors were blinded to the instructions and to the test results recorded by other assessors. Data were available for 137 observations, and these were dichotomized into either 'able to contract' ('correct contraction') or 'not able to contract' ('straining' or 'nothing'). This information was used to calculate the sensitivity, specificity and positive and negative predictive values for the CMT. Results: The CMT correctly identified 56/58 observations when women did not perform the contraction of the PFMs compared to $61 / 79$ observations when they did. Hence, the sensitivity and specificity were 97 and 77\%, respectively. Conclusion: This study showed that the CMT was a useful test to identify women who were able to localize their PFMs compared to those who were not, making it a potentially useful initial screening test in structured training programmes for the PFMs.

(c) 2014 S. Karger AG, Base

\section{Introduction}

Pelvic floor exercises are an important element in the prevention and treatment of pelvic floor dysfunction [1, 2]. Therefore, women are commonly advised, particularly at their first antenatal visit, to perform pelvic floor muscle training (PFMT) and to continue practising it thereafter. However, these instructions are seldom backed up by an objective test to ensure that the woman is able to locate her pelvic floor muscles (PFMs). Indeed, without guidance, more than $30 \%$ of women are unable to contract their PFMs correctly [3-5]. Several studies have concluded that PFMT requires proper instructions and an objective assessment to ensure muscle localization and also close follow-up $[1,6]$.

\begin{tabular}{ll}
\hline KARGER & $\begin{array}{l}\text { @ 2014 S. Karger AG, Basel } \\
1011-7571 / 14 / 0234-0318 \$ 39.50 / 0 \quad \text { Karger }\end{array}$ \\
E-Mail karger@karger.com & $\begin{array}{l}\text { This is an Open Access article licensed under the terms of the } \\
\text { Creative Commons Attribution-NonCommercial 3.0 Un- } \\
\text { ported license (CC BY-NC) (www.karger.com/OA-license), } \\
\text { applicable to the online version of the article only. Distribu- } \\
\text { tion permitted for non-commercial purposes only. }\end{array}$
\end{tabular}

Prof. Khaled M.K. Ismail, MSc, MD, PhD, FRCOG

Academic Unit, Birmingham Women's NHS Foundation Trust Edgbaston, Birmingham B15 2TG (UK)

E-Mailk.ismail@bham.ac.uk 

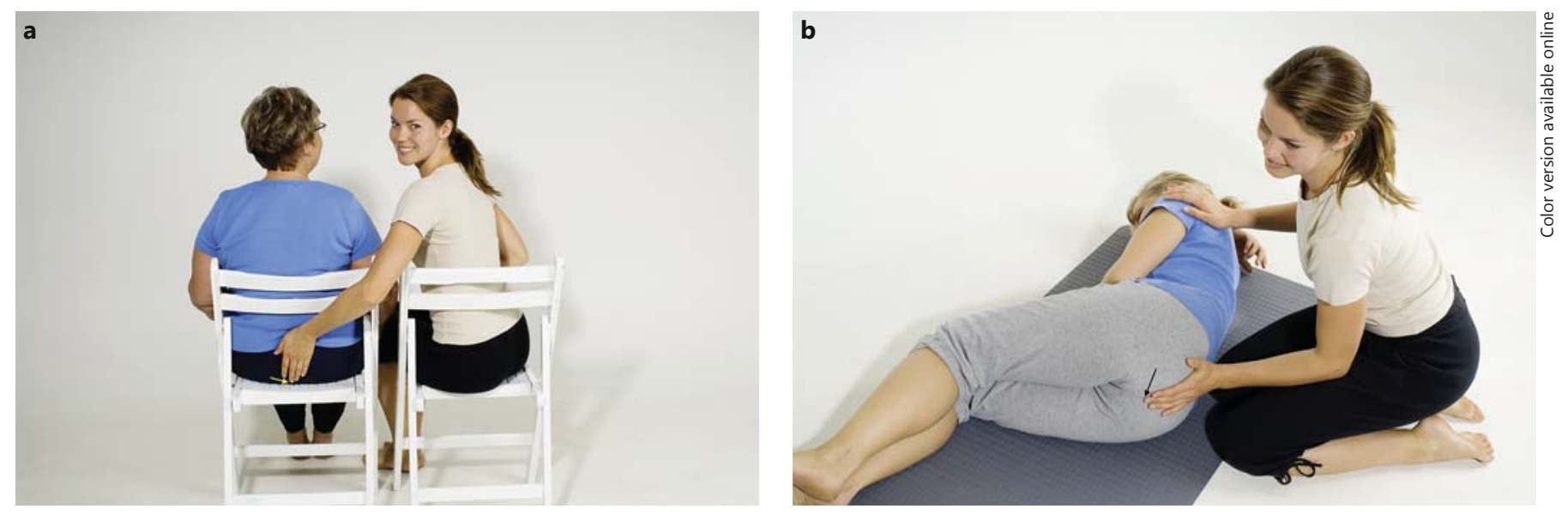

Fig. 1. The CMT performed in a sitting (a) and lateral (b) position.

The physical location of the PFMs and the setting in which preventive instructions are given often make it difficult to offer objective performance assessment and feedback to ensure that the exercises are performed correctly [7]. Moreover, when some women attempt to contract their PFMs, they hold their breath or brace their abdominal wall. These manoeuvres increase the intra-abdominal pressure and consequently depress the pelvic floor, which, in turn, weakens rather than strengthens the PFMs $[5,8,9]$. Interestingly, in this situation, the perceived upward movement of the perineum is actually a return of the pelvic floor from a downward to a resting position rather than an upward lift from the resting position, which can potentially affect the interpretation of some of the tests used to objectively assess PFM localization. It is therefore imperative to ensure that such tests can accurately differentiate between a perceived upward movement of the PFMs following an incorrect downward displacement compared to the true upward PFM lift secondary to a correct contraction, a 'squeeze' [9]. The muscular action involved in PFM contraction was first described by Kegel [10] in 1948, and has more recently been confirmed by ultrasound and dynamic magnetic resonance imaging studies of the pelvic floor. Bø et al. [11], using dynamic magnetic resonance imaging, showed that during PFM contraction, there was an inward lift of the levator plate with the coccyx moving in a ventral-cranial direction, whereas during straining, the coccyx was displaced in a caudal-dorsal direction. One of the authors (S.H.S.) proposed that a method of objective assessment of the ability to localize this group of muscles would be the coccygeal movement test (CMT) which can assess the presence and direction of coccygeal displacement whilst performing a PFM contraction.

The aim of this study was to evaluate the accuracy of the CMT as an objective and relatively non-invasive screening tool for the assessment of PFM localization.

\section{Subjects and Methods}

The study was approved by the Research Ethics Committee and a valid informed written consent was obtained from each participant prior to recruitment. Twenty-four healthy female volunteers known to be able to locate their PFMs upon vaginal examination (established by a qualified physiotherapist before enrolment) were recruited for this study. For each of the study participants, the weight in kilograms and height in centimetres were measured and the BMI was calculated.

Exclusion criteria were a history of neurological diseases, known pelvic floor dysfunction and the inability of women to locate their PFMs in the prerecruitment assessment or menstruating on the day of assessment. Participants were asked to draw an opaque, sealed envelope containing a note asking them to perform either a 'correct contraction', 'straining' or 'nothing' when assessed for their coccygeal movement. Forty envelopes ( 25 'correct contraction', 10 'straining' and 5 'nothing') were made available for use and were shuffled to ensure that the instructions were allocated at random. Each participant was assessed in a random order by six independent female physiotherapists. Participants were requested to wear soft material trousers and were asked to empty their urinary bladders prior to assessment.

In our experience, it is feasible to assess the CMT while the woman is in a standing, sitting or lateral position. In this study, the test was performed with the participant wearing light clothing and in the sitting position (for their convenience; fig. 1). The assessor placed the proximal part of their hand (thenar and hypothenar eminences) against the upper part of the woman's sacrum with the index and ring fingers on the gluteal muscles and the middle finger 
Table 1. Contingency table for CMT results (index test) against instructions (reference standard)

\begin{tabular}{llll}
\hline CMT & $\begin{array}{l}\text { Disease present } \\
\text { ('straining' or } \\
\text { 'nothing') }\end{array}$ & $\begin{array}{l}\text { Disease absent } \\
\text { ('correct } \\
\text { contraction') }\end{array}$ & Total \\
\hline $\begin{array}{l}\text { Positive } \\
\text { (no ventral } \\
\text { displacement) }\end{array}$ & 56 & 18 & 74 \\
$\begin{array}{l}\text { Negative } \\
\text { (ventral } \\
\text { displacement) }\end{array}$ & 2 & 61 & 63 \\
\hline Total & 58 & 79 & 137 \\
\hline
\end{tabular}

on or close to the coccyx. When the participant was asked to contract her PFMs, an inward displacement of the coccyx or the tissue around it was considered indicative of a 'correct contraction', while an outward or no displacement indicated 'straining' or 'nothing', respectively. The assessor gave the instruction 'squeeze' twice with no further instructions and otherwise did not talk to the participants during the assessment. However, the women had been previously advised to follow the same instruction provided in the 'note' every time they were assessed. The physiotherapists were asked to document whether the participant was either 'able' or 'not able' to perform a correct PFM contraction following each assessment. The assessors were blinded to the instructions received by the participants as well as the CMT results obtained by the other assessors. In this study, the presence of disease was defined as the inability to contract the PFMs. Similarly, the CMT was considered 'positive' if there was a perceived lack of ventral displacement upon coccygeal palpation (table 1). Complete datasets were available for 137 observations and were used for the final analysis. The information provided in the 'notes' was used as the reference standard following dichotomization of the information into 'able to contract' for the 'correct contraction' instruction or 'not able to contract' for either the 'straining' or 'nothing' instructions. All measurements were entered using EpiData. Analyses of data were performed using SPSS 11.0 statistical software. Results are given as 95\% confidence intervals. A significance level of 0.05 was chosen. Based on the number of true positive, true negative, false positive and false negative observations (table 1), the sensitivity, specificity and positive and negative predictive values for the CMT were calculated.

\section{Results}

The median age of participants was 57 years (range 24-80). Of the 24 participants, $15(62.5 \%)$ had a BMI within the range 18.5-24.9 and that of the remaining 9 (37.5\%) was in the range $25.0-29.9$. There was substantial interrater agreement in the observations between different assessors, with 117 (85\%) agreements observed (Co- hen's kappa coefficient $=0.71, \mathrm{SEM}=0.058,95 \%$ confidence interval 0.60-0.82).

The CMT correctly identified 56/58 observations when the women did not perform PFM contractions compared to $61 / 79$ observations when they did. Therefore, the accuracy of the CMT to identify problems in PFM localization had a sensitivity of $97 \%$, a specificity of $77 \%$, a positive predictive value of $76 \%$ and a negative predictive value of $97 \%$ (table 1 ).

\section{Discussion}

Based on this study, the CMT had better sensitivity (97\%) than specificity (77\%). It seemed to have a high negative predictive value, so the test could identify women who can locate their PFMs. In contrast, the lower positive predictive value would suggest that some women were falsely identified by the CMT as not able to locate their PFMs. This misidentification could potentially lead to some women having an unnecessary second confirmatory test, such as vaginal palpation. Nevertheless, without a prior CMT, all women would have needed to be assessed by vaginal palpation if we were to deliver a structured PFMT programme. Moreover, in the clinical setting, the assessors had the opportunity to do multiple testing while giving feedback, and this could potentially reduce false positive and false negative results.

A review by Mørkved and Bø [1] concluded that PFMT is indeed effective when it is supervised. Moreover, the latest Cochrane review of PFMT for both prevention and treatment of urinary incontinence in antenatal and postnatal women concluded that pregnant women without prior incontinence who were randomized to PFMT and supervision were less likely to report urinary incontinence up to 6 months after delivery than women randomized to no PFMT or usual antenatal care [12]. However, a randomized controlled trial failed to demonstrate the effectiveness of PFMT when exercises were taught in a general fitness class for pregnant women without individual instruction for correct contraction of the PFMs [13]. It would therefore be prudent if the availability of an objective assessment of the woman's ability to locate her PFMs were to form an integral part of any structured PFMT programme. Currently, the gold standard test used in the clinical setting is digital vaginal palpation; however, because of the nature of the test, whether it can be performed or not depends on the availability of a chaperone, the setting in which the PFMT is discussed and the acceptability of this test to the woman. This means that fre- 
quently women are advised to perform PFMT without offering them any objective assessment of whether or not they are able to locate their PFMs. Ensuring that women can localize their PFMs is a crucial component of any PFMT programme.

The CMT is a simple and less invasive test compared to vaginal examination and could prove beneficial as a screening tool to identify women who are unable to contract their PFMs. This could prove helpful, particularly during the antenatal period when community midwives routinely give instructions to women about pelvic floor exercises. In view of the setting in which these instructions are given and the reluctance of most women to have a vaginal examination in pregnancy, women are not objectively assessed with regard to their ability to locate their PFMs. We do not expect the CMT to replace vaginal palpation. Indeed, vaginal palpation should provide the additional benefit of assessing the strength of the contraction, although this aspect of the test has been reported to have suboptimal levels of reproducibility, sensitivity and validity [14]. However, similar to vaginal palpation, the CMT is a test proposed to assess the contraction of the PFMs and not their 'lifting' aspect. The latter is probably best assessed using dynamic magnetic resonance imaging [11]. The women in this study were assessed for their CMT in a sitting position where a degree of resting tone is to be expected [15]. It is possible that the presence of this tone would reduce the degree of coccygeal displacement with a contraction; hence the test accuracy could potentially improve if the woman is assessed in a lateral position where the PFMs would be in a more relaxed resting position.

There are several strengths to our study. We included only women who were able to locate and correctly contract their PFMs, while positive and negative reference test results were determined by instruction cards picked up at random by the participants. We attempted to minimize methodological biases by blinding the assessors to the participants' instructions, thus ensuring that the evaluated test (the CMT) and the reference standard were independent of each other. We also ensured that the physiotherapists were blinded to each other's assessments. The study also had limitations. Firstly, contraction of the abdominal wall muscles was not objectively assessed. In future studies, we suggest that the assessors do this by placing their hands on the woman's abdomen while performing the CMT. We intend to include this in any future studies evaluating the diagnostic accuracy of the CMT. Secondly, it is possible that the participants did not follow the card instructions every time they were assessed.

\section{Conclusion}

The CMT was a reproducible, easy-to-perform test that was useful for identifying women who can localize their PFMs. However, the lack of perceived movement on the CMT was not an accurate indicator of an inability to contract the right group of muscles. The CMT could potentially be a useful screening test to identify women who would benefit from a more structured approach for their PFMT.

\section{Acknowledgements}

The authors would like to thank the independent physiotherapists for their technical assistance (especially Pia Jørgensen, who conducted the vaginal palpations) and the women who participated in the study. The study was funded by the Association of Danish Physiotherapists Research Foundation and the Aarhus University Hospital Research Initiative.

\section{References}

1 Mørkved S, Bø K: Effect of pelvic floor muscle training during pregnancy and after childbirth on prevention and treatment of urinary incontinence: a systematic review. Br J Sports Med 2014;48:299-310.

2 Dumoulin C, Hay-Smith J: Pelvic floor muscle training versus no treatment, or inactive control treatments, for urinary incontinence in women. Cochrane Database Syst Rev 2010; 1:CD005654.
3 Benvenuti F, Caputo GM, Bandinelli S, et al: Reeducative treatment of female genuine stress incontinence. Am J Phys Med 1987;66: 155-168.

4 Bø K, Larsen S: Knowledge about and ability to correct pelvic floor muscle exercises in women with urinary incontinence. Neurourol Urodyn 1987;7:261-262.

5 Bump RC, Hurt WG, Fantl JA, et al: Assessment of Kegel pelvic muscle exercise performance after brief verbal instruction. Am J Obstet Gynecol 1991;165:322-327.
6 Bø K: Pelvic floor muscle training in treatment of female stress urinary incontinence, pelvic organ prolapse and sexual dysfunction. World J Urol 2012;30:437-443.

7 Mason L, Glenn S, Walton I, et al: The instruction in pelvic floor exercises provided to women during pregnancy or following delivery. Midwifery 2001;17:55-64. 
8 Thompson JA, O’Sullivan PB, Briffa NK, et al: Assessment of voluntary pelvic floor muscle contraction in continent and incontinent women using transperineal ultrasound, manual muscle testing and vaginal squeeze pressure measurements. Int Urogynecol J Pelvic Floor Dysfunct 2006;17:624-630.

-9 Bø K, Sherburn M: Evaluation of female pelvic-floor muscle function and strength. Phys Ther 2005;85:269-282.
10 Kegel AH: The nonsurgical treatment of genital relaxation; use of the perineometer as an aid in restoring anatomic and functional structure. Ann West Med Surg 1948;2:213216.

11 Bø K, Lilleas F, Talseth T, et al: Dynamic MRI of the pelvic floor muscles in an upright sitting position. Neurourol Urodyn 2001;20: 167-174.

12 Boyle R, Hay-Smith EJC, Cody JD, et al: Pelvic floor muscle training for prevention and treatment of urinary and faecal incontinence in antenatal and postnatal women. Cochrane Database Syst Rev 2012;10:CD007471.
$13 \mathrm{~B} ø \mathrm{~K}$, Haakstad LA: Is pelvic floor muscle training effective when taught in a general fitness class in pregnancy? A randomised controlled trial. Physiotherapy 2011;97:190-195.

-14 Bø K, Finckenhagen HB: Vaginal palpation of pelvic floor muscle strength: inter-test reproducibility and comparison between palpation and vaginal squeeze pressure. Acta Obstet Gynecol Scand 2001;80:883-887.

$15 \mathrm{~B} ø$ K, Finckenhagen HB: Is there any difference in measurement of pelvic floor muscle strength in supine and standing position? Acta Obstet Gynecol Scand 2003;82:11201124. 